\title{
In vivo metabolomic study uncovers distinct metabolic phenotypes of host tissues and predicts oxidative state of acute myeloid leukemia
}

\author{
Guillaume Cognet \\ INSERM \\ Lucille Stuani \\ Inserm \\ Thomas Farge \\ Inserm \\ Mathilde Gotanègre \\ Inserm \\ Maud Heuillet \\ INSA Toulouse \\ Lara Gales \\ INSA Toulouse \\ Amandine Rocher \\ INSA Toulouse
}

Nina Lager-Lachaud

INSA Toulouse

Claudie Bosc

Inserm

Marie Sabatier

Inserm

Estelle Saland

Inserm

Ambrine Sahal

INserm

Laura Poillet-Perez

Inserm

Francois Vergez

Inserm

Véronique de Mas

Inserm

Christian Récher 
Inserm

Jean-Charles Portais

INSA Toulouse

\section{Floriant Bellvert}

INSA Toulouse

Jean-Emmanuel Sarry ( $\sim$ jean-emmanuel.sarry@inserm.fr )

INSERM Délégation régionale Midi-Pyrenees Limousin https://orcid.org/0000-0002-6704-2032

\section{Research}

Keywords: in vivo metabolomics, metabolic niche, OxPHOS state, prediction, leukemia

Posted Date: July 20th, 2021

DOI: https://doi.org/10.21203/rs.3.rs-719532/v1

License: (c) (i) This work is licensed under a Creative Commons Attribution 4.0 International License. Read Full License 


\section{Abstract \\ Background}

Metabolic adaptation is a hallmark of cancer including acute myeloid leukemia (AML). Tumor microenvironment is also described as an essential support of leukemic metabolism. We explored how systemic and tissue metabolism was rewired in leukemia-bearing mice and upon chemotherapy.

\section{Methods}

Using AML cell line- and primary patient-derived xenograft models, we developed in vivo metabolomics to uncover the metabolic pattern of 10 tissues including plasma, bone marrow, spleen, liver, adipose tissue, lung, pancreas, kidney, heart and muscle.

\section{Results}

In vivo targeted mass spectrometry allowed metabolic characterization of tissues from naïve and AMLxenografted immunocompromised mice. AML xenotransplantation and cytarabine treatment induced AML cell type-dependent global changes in tissue metabolomes. Infiltration of high OxPHOS MOLM14 cells that are intrinsically chemoresistant, induced minor changes in tissue metabolomes. In contrast, low OxPHOS U937 xenograft led to major reprogramming of metabolic tissue niches for survival upon chemotherapy. Interestingly, plasma metabolite signatures could predict the oxidative phenotype of leukemic cells.

\section{Conclusion}

Major metabolic changes in host tissues play a crucial role in tumor xenotransplantation and define their OxPHOS state in AML. Since mitochondrial phenotype is an essential determinant of drug response in AML, plasma metabolite signatures might be novel biomarkers for patient stratification.

\section{Background}

Since the discovery of the Warburg Effect, it was widely considered that cancer cells sustain reprogrammed metabolism, allowing them to face stressful challenges such as hypoxia, chemotherapy or metastasis $^{1-3}$. In this context, we and others have shown that enhanced oxidative phosphorylation (OxPHOS) activity and mitochondrial adaptation are major components of drug response in multiple cancers including acute myeloid leukemia $(\mathrm{AML})^{4-8}$. The availability of various nutrients is needed to support this enhanced mitochondrial activity. Tumor microenvironments play a crucial role in this process by supplying cancer cells with glucose, amino acids and fatty acids ${ }^{9-15}$. In AML, leukemic cells are able 
to migrate through diverse microenvironments and colonize chemoprotective niches, other than hematopoietic organs, leading to the emergence of extramedullary disease (EMD), metastatic-like situations and poor prognosis ${ }^{16-19}$.

To study the role of the tumor microenvironment into metabolic adaptations, mouse models are widely used $^{20}$. They provide the systemic environment essential for better understanding of cancer biological characteristics such as engraftment, angiogenesis, metastasis, immunoresponse or drug systemic metabolization ${ }^{21-23}$. From transgenic mice harboring murine cancer to humanized mice, many models have been developed. One of the most frequently used murine model is the highly immunodeficient NOD/SCID-IL2rg (NSG) mice xenografted with cell lines or primary cells ${ }^{24-27}$. Due to its enhanced immunodeficiency, this model allows optimal engraftment and proliferation of human cancer cells ${ }^{26}$. This model has been exhaustively described from an anatomic, histologic and hematological point of $v_{i e w}{ }^{23,28}$. Surprisingly, to our knowledge, little is known about metabolic profiles of tissues from diverse murine strains including NSG. Only one study described tissue metabolomes from two naïve male C57BL/ $6^{29}$, and several works showed tissue metabolic rewiring ${ }^{13,30,31}$ and plasma metabolomes ${ }^{13,32}$.

Furthermore, the impact of metabolite availability on studied phenotypes has become a major question in metabolomic studies ${ }^{33-36}$. For instance, uric acid was found to have a critical role in the inhibition of in vivo 5 -fluoroacid treatment on sarcomas ${ }^{36}$. While this metabolite is abundant in human plasma, it is not found in murine models, and the study of this treatment has strong limitations in vivo. Thus, describing the metabolic state of naive mouse niches is essential for the complete understanding of metabolic reprogramming and flexibility of cancer cells in vivo.

Here, we described metabolic profiles of polar metabolites from ten tissues of interest (i.e. plasma, bone marrow, spleen, liver, subcutaneous white adipose tissue or SWAT, lung, pancreas, kidney, heart and muscle) in naïve NSG mice. We further showed changes in tissue metabolism induced by either leukemic engraftment or cytarabine (AraC) treatment, and demonstrated that metabolic features of injected AML cells were drivers of tissue metabolic rewiring in vivo. We also unraveled plasma signatures of the metabolic state of AML cells. Altogether, this study provides knowledge about metabolic profiles of NSG mice tissues and a better understanding of the systemic metabolic reprogramming following leukemic engraftment and AraC treatment. Finally, our results proposed plasma metabolic signature of AML mitochondrial state as novel biomarkers predictive of drug response in AML.

\section{Methods}

Primary samples and ethical statement. Primary samples were isolated from patient peripheral blood by Ficoll gradient separation and stock in liquid nitrogen until further use. Primary AML patient cells from peripheral blood have been collected during routine diagnostic procedures at the Toulouse University Hospital (TUH), after informed consent and stored at the HIMIP collection (BB-0033-00060). According to the French law, HIMIP collection has been declared to the Ministry of Higher Education and Research (DC 
2008 - 307 collection 1) and obtained a transfer agreement (AC 2008 - 129) after approbation by the "Comité de Protection des Personnes Sud-Ouest et Outremer II" (ethical committee). Clinical and biological annotations of the samples have been declared to the CNIL ("Comité National Informatique et Libertés"; i.e. "Data processing and Liberties National Committee"). Peripheral blood and bone marrow samples were frozen in fetal calf serum with $10 \%$ DMSO and stored in liquid nitrogen.

Mice and mouse xenograft model. Animals were used in compliance with a protocol reviewed and approved by the Institutional Animal Care and Use Committee of Région Midi-Pyrénées (France). NOD/LtSz-SCID/IL-2Rychainnull (NSG) mice were produced at the Genotoul Anexplo platform at Toulouse (France) using breeders obtained from Charles River Laboratories. Mice were housed in sterile conditions using high-efficiency particulate arrestance filtered microisolators and fed with irradiated food and sterile water. AML cell lines and human primary AML cells were transplanted as reported previously ${ }^{4,24}$. Briefly, mice (7-8 weeks old) were sublethally treated with busulfan $(20 \mathrm{mg} / \mathrm{kg}) 24$ hours

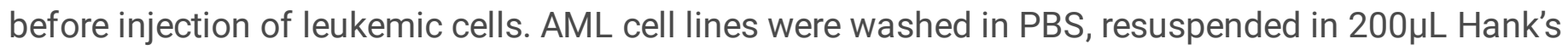
Balanced Salt Solution (HBSS) at a final concentration of 0.5 and $2 \times 10^{6}$ cells (for resp. U937 and MOLM14) and injected into tail vein.

For leukemia patient samples, cells were thawed at room temperature, washed twice in PBS, resuspended in $200 \mu \mathrm{L} \mathrm{HBSS}$ at a final concentration of $0.2-10 \times 10^{6}$ cells and injected into tail vein. Transplanted mice were treated with antibiotic (Baytril) for the duration of the experiment. Daily monitoring of mice for symptoms of disease (ruffled coat, hunched back, weakness, and reduced mobility) determined the time of killing for injected animals with signs of distress. If no signs of distress were seen, mice were considered engrafted after a week for cell lines or were initially analyzed for engraftment after 8 weeks for patient samples except specified otherwise.

In vivo mice treatment. After engraftment (1 week for cell line-derived xenografts or CLDX; 8 to 18 weeks as determined by flow cytometry on peripheral blood or bone marrow aspirates for patient-derived xenografts or PDX), NSG mice were treated with AraC. NSG mice were treated by daily intraperitoneal injection of $30 \mathrm{mg} / \mathrm{kg} \mathrm{AraC} \mathrm{(} 60 \mathrm{mg} / \mathrm{kg}$ for PDX) for 5 days. AraC was kindly provided by the pharmacy of the TUH. For control, NSG mice were treated daily with intraperitoneal injection of vehicle, PBS 1X. Mice were monitored for toxicity and provided nutritional supplements as needed.

Assessment of leukemic engraftment. Assessment of leukemic engraftment was measured as reported previously. Briefly, NSG mice were humanely killed in compliance with European ethics protocols. Bone marrow (mixed from tibias and femurs) and spleen were dissected in a sterile environment and flushed in HBSS with $1 \% \mathrm{FBS}$. Mononuclear cells isolated by tissue lysis with $\mathrm{NH}_{4} \mathrm{Cl}$ from bone marrow, and spleen were labeled with hCD33-PE (555450), mCD45.1-PerCP-Cy5.5 (156058), hCD45-APC (5555485), and hCD44-PECy (7560533) (all antibodies from BD Biosciences) to determine the fraction of human blasts (hCD 45 + mCD45.1 - hCD33 + hCD44 + cells) using flow cytometry. All antibodies used for cytometry were used at concentrations between $1 / 50$ and 1/200 depending on specificity and cell density. Analyses were performed on a CytoFLEX flow cytometer with CytoExpert software (Beckman Coulter) and FlowJo 10.2 
(Tree Star). The number of AML cells/ $\mu$ l peripheral blood and number of AML cells in total cell tumor burden (in bone marrow and spleen) were determined by using CountBright beads (Invitrogen) using described protocol.

Tissue sampling for metabolomics. Plasma samples were isolated from peripheral blood of mice, collected prior to the sacrifice. After centrifugation (@3000rpm, 5min, @ $4^{\circ} \mathrm{C}$ ), plasma supernatant was collected and fast-frozen in liquid nitrogen.

Tissues samples were collected in this order: spleen, pancreas, kidney, liver, lung, heart, subcutaneous adipose tissue, muscle and bone marrow. After weighting, all tissues were fast-frozen in liquid nitrogen. Spleen and bone marrow samples were halved in order to simultaneously perform the leukemic engraftment analysis. Tissue collection from mouse sacrifice to fast-freezing was done within 5 minutes. All samples were stored at $-80^{\circ} \mathrm{C}$ until further use.

Metabolite profiling. A volume of $10-50 \mu \mathrm{L}$ of plasma were thawed for extraction. Milli-Q water was added q.s. $100 \mu \mathrm{L} .400 \mu \mathrm{L}$ of cold methanol were then added for extracellular matrix precipitation. After thorough mixing and incubation at $-20^{\circ} \mathrm{C}$ for $15 \mathrm{~min}$, samples were centrifuged (@2000g, 5min, @ $4^{\circ} \mathrm{C}$ ) and supernatant was recovered. $35 \mu \mathrm{L}$ of isotope dilution method standard solution ${ }^{37}$ was added to $125 \mu \mathrm{L}$ of extract, dried using the Eppendorf Concentrator 5301 and resuspended into $60 \mu \mathrm{L} \mathrm{Milli-Q}$ water. Two 30 $\mu \mathrm{L}$ vials were prepared for central metabolites and amino acids analyses.

Solid tissues were first homogenized in cold conditions using a tissue lyser, with three 1-minute cycles at $25 \mathrm{~Hz}$ and $5-10 \mathrm{mg}$ of homogenized tissue were weighed. $750 \mu \mathrm{L}$ of acetonitrile/methanol/Milli-Q water $(2: 2: 1)+0.1 \%$ formic acid were added to the samples. The resulting pellets were then lysed with two 1minute cycles at $25 \mathrm{~Hz}$. After incubation at $-20^{\circ} \mathrm{C}$ for $15 \mathrm{~min}$, samples were centrifuged (@2000g, 5min, $@ 4^{\circ} \mathrm{C}$ ) and supernatant was recovered. Isotope dilution method standard solution was added to the extract $(35 \mu \mathrm{L}$ for $1000 \mu \mathrm{L}$ of extract for central metabolites analysis; $25 \mu \mathrm{L}$ for $75 \mu \mathrm{L}$ of extract for amino acids analysis), dried using the Eppendorf Concentrator 5301 and resuspended into 50 $\mu \mathrm{L}$ Milli-Q water. Metabolites were quantified using methods described previously ${ }^{38}$, using the TraceFinder 4.1 software, operating under Xcalibur 4.1 (Thermo Fisher Scientific, Austin, TX).

Single sample Gene Set Enrichment Analysis (ssGSEA). The transcriptomes of 35 primary samples were analyzed using the ssGSEA module of the GenePattern website ${ }^{39}$. Obtained enrichment scores for OXPHOS signatures were normalized with a z-score.

Principal Component Analysis (PCA). Plasma datasets of metabolite concentrations were normalized to the naïve mice plasma and transformed into logarithm 10. The PCA was computed using the R package mixOmics ${ }^{40}$.

Statistical analyses. Statistical analyses were conducted using Prism software v9.0 (GraphPad Software, La Jolla, CA, USA). Statistical significance was determined by the two-tailed unpaired Student's t-test. A pvalue $<0.05$ was considered statistically significant. For all figures, ns- not significant, *p\% 0.05 , 
$\star \star x p 0.01, * \star \star p \% 0.001, \star \star \star \star x \% 0.0001$. Unless otherwise indicated, all data represent the mean \pm standard error of the mean (SEM) from at least three independent experiments.

\section{Results}

Metabolic pattern is markedly different between the tissues of naive NSG mice. To understand how the xenotransplantation of human AML cells rewire tissue metabolism, we first characterized the metabolic profiles of ten different tissues (plasma, bone marrow (BM), spleen (SP), liver, subcutaneous white adipose tissue (SWAT), pancreas, lung, kidney, heart and muscle) from 4 naïve 8 weeks-old male and female NSG mice (Fig. 1a). Using two complementary LC-MS methods, 48 metabolites were quantified in absolute or relative amounts in each tissue. The measured metabolites were representative of the major metabolite classes (e.g. phosphorylated sugars, organic acids, amino acids (AA), nucleotides), and covered central and energy metabolic pathways (glycolysis, pentoses-phosphate pathway, TCA cycle and related processes, amino acid metabolism, nucleotide metabolism). Metabolic profiles of plasma from the naïve mice showed high levels of amino acids (range from 0.1 to $1 \mathrm{mM}$ ), especially valine, glutamine, glycine and alanine. AA are key components of metabolism, as they constitute the main product of digested proteins and can be fuels for energy and biomass production. In contrast, other metabolites (glycolytic and TCA cycle intermediates, nucleotides and phosphorylated sugars) were observed in lower concentrations (range from 0.1 to $1.0 \mathrm{mM}$ ) (Fig. 1b). We were able to compare AA concentrations in NSG plasma with the data found in the $\mathrm{MMMDB}^{29}$ and observed that they were found in similar ranges. Other metabolites could not be compared.

Due to the vast range of concentrations in central metabolites in the different solid tissues, we normalized metabolite concentrations using a z-score (Fig. 1C). AA absolute amounts were also graphed in the different tissues (Additional Fig. 1a). Overall, most tissues had similar profiles, with the exception of two peculiar tissues (SWATs and SP). SWATs were characterized by the lowest metabolites concentrations and SP showed high levels in AA, early glycolytic intermediates and phosphorylated sugars. Low amounts of metabolites were expected in SWATs, as they are the major lipid reservoir. Surprisingly, AA and glycolytic intermediates in SP was over-abundant in this tissue. The SP is a hematopoietic organ with cellular composition similar to that of the BM and the overabundance of nutrients in this organ might explain the high propensity of leukemic cells to engraft this tissue. Because of the differences in AA content in SP, we further assessed AA distribution and concentration in all tissues (Fig. 1d). AA distribution was found quite similar in most tissues, with the exception of SWATs, showing undetectable amounts of glycine, and plasma, showing the lowest amounts of aspartate. This indicates that proteinogenic amino acids balance is conserved in the different organs.

Since gender is one of intrinsic factor regulating host metabolism, we also wanted to evaluate the impact of sex on metabolic profiles (Additional Fig. 1b). To our knowledge, no study was done on this aspect, and significant changes could bias further results. Interestingly, we found very few sex-specific changes in metabolite quantities in most tissues. The most impacted tissue was the BM with 6 amino acids (arginine, leucine, phenylalanine, serine, tyrosine and valine) significantly increased in female mice. We 
concluded that sex-driven changes were minimal in this cohort. Indeed, we studied 7-8 weeks-aged animals. These mice were at an early stage of puberty, indicating that sex-dependent metabolic impacts were minimized. Knowing that mice life span can extend to at least one year after cell engraftment for patient-derived xenografts, sex-driven metabolic changes would be uncovered at later stages.

\section{AML xenotransplantation induces a profound reprogramming of local and systemic metabolism in vivo.}

We investigated the impact of leukemic engraftment on murine tissue metabolic profiles. NSG mice were engrafted with two metabolically different AML cell lines: high OxPHOS AraC-resistant MOLM14 cells and low OxPHOS AraC-sensitive U937 cells ${ }^{4}$. We have previously shown that the distribution of AML cells was variable in hematopoietic tissues of NSG mice post-transplantation ${ }^{24}$. MOLM14 and U937 cells mostly invade BM and SP, respectively. After two weeks of transplantation, we sacrificed mice and assessed leukemic engraftment in these two hematopoietic tissues by flow cytometry (Additional Fig. 2a-c). We confirmed significant differences in leukemic infiltration between MOLM14 and U937 cells. Other organs were not analyzed by cytometry, and possible engraftment could not be assessed. Then, metabolic profiles were done for the 9 solid tissues (Fig. 2a).

Metabolome of MOLM14-engrafted tissues showed little changes compared to their respective naïve metabolome. 24 metabolites were differentially changed mostly in BM (Fig. 2b). Asparagine, aspartate and phosphoenolpyruvate were depleted in MOLM14-engrafted mice, whereas glutamate, UMP, CMP, 2hydroxyglutarate, galactosamine-1-phosphate (GalN-1P), N-acetylglucosamine-1-phosphate (GIcNAc-1P) and $\mathrm{N}$-acetylglucosamine-6-phosphate (GIcNAc-6P) were accumulated. Changes were also observed in muscles with depletion of CMP, GMP, alanine, glutamate, glutamine, and pentoses-5-phosphate, in the pancreas with the depletion of GMP and pentoses-5-phosphate, in kidney with the depletion of glutamine and accumulation of ribose-1-phosphate, in the lung with an accumulation of UTP and glutamine, and in the heart with an accumulation of lactate and pentoses-5-phosphate (Fig. 2a).

On the other hand, U937 engraftment led to major changes in metabolic profiles compared to naïve tissue metabolome (Fig. 2c). Lung was the most impacted tissue with depletion of aspartate and glycine, and accumulation of AAs, fructose-1.6-biphosphate (FBP), fructose-6-phosphate (Fru6P), 2-hydroxyglutarate $(2-H G)$, and derivatives of uridine. BM was characterized by depletion of aspartate, asparagine, glycine, GalN-1P and GIcNAc-1P, and accumulation of pyruvate, CMP and 2-HG from U937-xenografted mice. SP was characterized with depletion of aspartate, asparagine, serine, methionine, orotate, glucose-6phosphate and Fru6P. Liver showed depletion of succinate and GalN-1P, and accumulation of glutamate, phenylalanine and threonine, while pancreas was characterized by depletion of glutamine and sedoheptulose-7-phosphate (Sed7P), and accumulation of glutamate, branched chained AA (BCAA), tyrosine, phenylalanine and citrate \& isocitrate. Pentose-5-phosphates, CMP and glutamate were depleted, and BCAA accumulated in muscles. Heart was significantly exhibited a depletion of serine, and an accumulation of BCAA, phenylalanine, 2-hydroxyglutarate, Sed7P and GIcNAc-6P. Finally, kidney showed depletion of glycine and alanine, and accumulation of UDP (Fig. 2c). 
Importantly, comparing global metabolic profiles after the xenotransplantation of both AML cell lines in vivo showed the most changes (Fig. 2d). In brief, AA were accumulated in SP and kidneys of MOLM14engrafted mice, whereas they were accumulated in the lungs, SWAT and pancreas of U937-engrafted mice. Glycolytic intermediates were accumulated in SP of MOLM14 xenografts and accumulated in lungs of U937 xenografts. This suggests that the metabolic impact of AML engraftment is mostly driven by the intrinsic characteristics of injected AML cells and their organ distribution (Fig. 2d). Of note, we also showed commonly affected metabolites in hematological tissues of both xenografts (BM of MOLM14 xenografted mice (Fig. 2e), and in both the BM and SP of U937 xenografted mice (Fig. 2f, 2g)). Aspartate, asparagine and orotate were depleted, whereas UMP and CMP were found accumulated in U937xenografted tissues. Interestingly, these changes were not found in SP of MOLM14 CLDX (Fig. 2h). Since engraftment of MOLM14 cells in SP is too small (Additional Fig. 2b), this could indicate that this common modulation is in a blast-dependent manner. These biggest changes in metabolic pathways suggest a rewiring of aspartate metabolism in the nucleotide biosynthesis (Fig. 2i).

Cytarabine treatment modifies systemic metabolism in AML xenografted mice. To understand how chemotherapeutic agents might affect tissue metabolism of AML-engrafted mice, we treated mice with $\operatorname{AraC}(30 \mathrm{mg} / \mathrm{kg} / \mathrm{d}$ ), or its vehicle PBS for a week (Additional Fig. 2a). As previously described, chemotherapy did not induce any significant reduction of the total cell tumor burden in MOLM14xenografted model, while it significantly reduced engraftment of U937 cells (fold-change of 13 and 276 in BM and SP, respectively; Additional Fig. 2b-c). Metabolic profiles of the 9 tissues of xenografted mice treated with AraC were performed as described above, with a normalization to the PBS-treated conditions (Fig. 3a).

MOLM14-engrafted mice treated with AraC showed modest modulations compared to PBS treatment (Fig. 3b). Those changes were mostly observed in SP with a net accumulation of AA after treatment (Fig. 3a). Lactate was increased after treatment in both SP and liver, and some nucleotides-related metabolites (orotate, cytidine monophosphate, guanosine diphosphate, and uridine triphosphate) were modulated in BM, SP, pancreas and heart. This absence of global changes in tissue metabolomes after AraC treatment was also observed when comparing naïve mice and AraC-treated MOLM14-engrafted mice (Fig. 3c). In addition, metabolic changes were similar to the one observed by comparing vehicletreated mice to naïve NSG (Fig. 3c versus Fig. 2b). However, additional changes were observed with an accumulation of galactosamine-1-phosphate (SP and lung), of orotate (kidney), and UDP (muscle), of BCAA (isoleucine, valine and phenylalanine), and pyrimidines (CMP and UMP) in heart.

Importantly, AraC-treated U937-xenografted mice were characterized by major metabolic changes (Fig. 3d). AA were globally accumulated in SP and liver, and depleted in pancreas and muscle. Some common difference in AA were seen in most organs, with an accumulation of aspartate, glutamate, glycine and serine. These AA can all fuel glutathione synthesis for reactive oxygen species (ROS) detoxification, suggesting that AraC treatment on this model globally impact ROS production and antioxidant defense. Glycolytic intermediates and phosphorylated sugars were accumulated in both muscle and lung. TCA cycle intermediates amounts were globally unchanged, with the exception of liver. In this 
tissue, succinate, fumarate and malate were accumulated, suggesting that AA such as glutamine, glutamate and aspartate were more fueled into energy production. Lastly, nucleotides were globally depleted in lung. All these changes led towards a 'naïve-like' condition, as tumor burden was greatly decreased upon AraC treatment (Additional Fig. 2b-c). When comparing AraC-treated U937-engrafted mice with naïve ones (Fig. 3e), most changes were reversed, with the exception of phosphorylated sugars, being depleted in SP and accumulated in BM and lung.

Of note, orotate pool was recovered in BM of both xenografted models and in SP of U937-engrafted mice post-AraC (Fig. 3f). AraC-treatment widely impact the energy metabolism and $\mathrm{OxPHOS}^{4}$, and dihydroorotate dehydrogenase (DHODH) one of the enzymes involved in the biosynthesis of nucleotides from aspartate is also dependent on the electron flux by reduction of ubiquinone ${ }^{41}$. Accordingly, orotate accumulation could imply an increase of the electron transfer chain activity in the whole tissue. Interestingly, orotate was detected in two other organs, lung and kidney, and no significant changes were seen in these organs (Additional Fig. 2d). Therefore, this phenotype is only found in engrafted hematological tissues. Altogether, we proposed that intrinsically chemoresistant MOLM14 cells do not induce global change of tissue metabolomes, whereas U937 significantly undergo a massive decrease in tumor burden with a profound reprogramming in metabolomes back to a 'naïve-like' state.

\section{Plasma LC-MS profiling experiment provides metabolic biomarkers of the oxidative phenotype of tumor in} vivo. To assess whether plasma metabolome might reflect metabolic signature of specific host tissues or/and AML, metabolic profiling of plasma was performed as described above for tissues (Fig. 4a). Except AA, level of all studied metabolites was changed post-xenotransplantation. TCA cycle intermediates were accumulated in MOLM14 xenograft and depleted in U937 xenograft. Glycolytic intermediates, nucleotides-related, and phosphorylated sugars were accumulated in U937 xenograft and depleted in MOLM14 xenograft. Since U937 and MOLM14 cells displays low and high OxPHOS dependency ${ }^{4}$, we hypothesized that plasma metabolic signatures might predict the oxidative state of AML tumors. We thus performed an unsupervised principal component analysis (PCA) of metabolomic data in the five conditions (naïve, MOLM14, U937, PBS or AraC; Fig. 4b), and found that $54 \%$ of the overall variation in plasma metabolic profiles allowed significant discrimination of the two types of AML xenograft with several metabolites as representative of each xenograft (Fig. 4a). These results suggest a specific signature of central metabolites as biomarkers of the metabolic state of the injected/xenografted AML cells.

To further explore this assumption, we analyzed a second independent cohort of mice (cohort 2), including 3 CLDX models (MOLM14, U937 or KG1a, another low OxPHOS AML cell line), and 8 different patient-derived xenograft (PDX) models (IM06, IM26, IM31, IM76, IM110, Ps325, Ps 6030 and Ps6312; Additional Table 2). Based on the transcriptome of primary patient AML blasts, the oxidative status of 4 PDX models (IM06, IM31, IM76 and Ps325) was characterized by single sample gene set enrichment analysis (ssGSEA) using curated OxPHOS gene signatures (Fig. 4c). Accordingly, Ps325 and IM31 were found to have a low OxPHOS status, whereas IM06 and IM76 display a high OXPHOS status. The plasma metabolic profiles of the cohort 2 were performed, and the results were normalized to the naive state as 
described in our first cohort (Fig. 4d). We then performed PCA on CLDX and PDX samples with known OxPHOS status, and found that $20 \%$ of the global variation allowed discrimination of plasma metabolomes according to the OxPHOS status of injected cells (Fig. 5e). When comparing the contributing metabolites for low and high OxPHOS status in both cohorts (Fig. 5f), we found 13 common metabolites accumulated in low OxPHOS CLDX and PDX models, including 2.3-diphosphoglycerate, 6phosphogluconate, adenine mono/di/triphosphate, fructose-6-phosphate, guanosine mono-/diphosphate, glucose-6-phosphate, mannose-6-phosphate, pentoses-5-phosphate and uridine mono/diphosphate. We also found 6 common metabolites accumulated in high OxPHOS CLDX and PDX models, including arginine, citrate/isocitrate, fumarate, lactate, malate and pyruvate. This indicates that this set of murine plasma metabolites might be predictive of the OxPHOS status of tumor cells.

\section{Discussion}

In this study, we aimed to study metabolic profiles of engrafted NSG mice tissues. We found two adaptive profiles specific of the engraftment and treatment. AraC-treated MOLM14 xenograft induced minimal changes in tissue metabolomes as similar as the untreated xenograft. This confirmed that MOLM14 cells are intrinsically primed for $\mathrm{AraC}$ resistance and they do not induce a global redirection of host metabolism to survive upon AraC treatment. As opposed, tissues of AraC-treated sensitive U937xenografted mice underwent major metabolic reprogramming. This indicates that U937 residual cells largely rewire the host metabolism to sustain their proliferation and adaptation to AraC treatment. Glycolysis and energetic metabolism were changed in hematopoietic niches, suggesting an increase in the OxPHOS status in the murine microenvironment of these niches. Further, AraC treatment induced accumulation of methionine, serine and glycine in most organs of U937-xenografted mice. These three metabolites are mainly involved in ROS management through glutathione biosynthesis, folate cycle and transulfuration pathway. It is known that AraC leads to an increase in ROS content in vivo, through enhanced OxPHOS status ${ }^{4,42}$. An increase in these three metabolites in most organs support an adaptation of murine niches to this oxidative stress. Finally, we observed a depletion in glucose-6phosphate, fructose-6-phosphate and mannose-6-phosphate after U937 engraftment in highly glycolytic muscle tissues. This suggest that glucose utilization in the muscle is highly diminished and hijacked by glycolytic U937 cells. This is consistent with another study that described the capacity of leukemic cells to rewire systemic glucose utilization through enhanced insulin resistance ${ }^{9}$, and suggests that highly glycolytic U937 cells are able to compete with distant muscle tissue for glucose utilization.

Changes in aspartate metabolism and nucleotide production were found common to both CLDX models. Aspartate, asparagine, orotate and phosphoenolpyruvate pools tended to be depleted, whereas CMP and UMP are increased in engrafted hematological tissues. This suggests that aspartate is specifically rewired in engrafted hematopoietic organs to sustain nucleotide production and proliferation as previously documented ${ }^{13}$. AraC treatment leads to replenishment of the orotate pool, favored by the enhanced $\mathrm{OxPHOS}$ status ${ }^{4,13}$. Having a higher OxPHOS status upon treatment leads to accumulation of reduced ubiquinone in the electron transfer chain, which could be used for the repletion of the orotate 
pool, through the activity of mitochondrially bound DHODH. This could facilitate the proliferation of residual cells and relapse. DHODH inhibitors such as Brequinar are already being evaluated in clinical trials alone (NCT03760666), and mechanisms of action have already been published ${ }^{43}$. Our study supports the rationale of a combination of AraC and DHODH inhibitor to slow down the appearance of relapses.

Importantly, we found that a set of plasma metabolites, including TCA cycle and glycolysis intermediates, could discriminate AML cells in vivo based on their oxidative status. While this metabolite set explains the half of the variations in our feasibility analysis (with MOLM14 and U937 xenografts), it only explains a fifth of the variation in second cohort (multiple CLDX and PDX models). Patient AML cells have a high genetic and metabolic heterogeneity ${ }^{44}$. CLDX and PDX engraftment time and tissue distribution can also widely vary ${ }^{24,45}$. Thus, the finding of a common signature efficiently discriminating injected cells depending on their OxPHOS status is promising. Since OxPHOS status is tightly related to chemoresistance, plasma metabolic signatures might also represent relevant metabolic biomarkers for drug response in AML. Interestingly, previous work has shown that glycerol-3-phosphate, pyruvate, lactate, citrate, 2-HG and alpha-ketoglutarate concentrations in patient plasma constitute an independent factor of low response to AraC treatment ${ }^{46}$. Most of these metabolites are markedly impactful in our metabolic signature from human patient plasma. The OxPHOS status is described as critical to many drug resistances in $A M L^{4,11,42}$. Accordingly, plasma is a tissue of interest for biomarker of the oxidative status of patient blasts to improve management of AML patients.

However, several limitations to our study should be taken into consideration, the main one being that mice were injected intravenously. The developed disease does not completely reconstitute leukemic appearance in patients. Some observed changes could be due to non-relevant colonization of distant tissues. Furthermore, our metabolomic studies were performed on bulk tissues. This did not distinguish metabolic changes in leukemic cells from those in the murine microenvironment within each tissue. Interstitial fluids could not be distinguished from the cells. Thus, metabolite amounts in naïve mice tissues do not correspond to the bioavailable amounts for leukemic cells. Techniques for interstitial fluid isolation are emerging and could be greatly useful for better understanding of dialogues between microenvironment and leukemic cells ${ }^{13,47}$. A study of leukemic and microenvironmental sorted cells from these tissues could lead to further rewiring and dialogues observations that could be exploited for therapy. Finally, techniques such as single cell metabolomics (SCM $)^{48}$ or mass spectrometry imaging $(\mathrm{MSI})^{49,50}$ could be useful, although they are not quite adapted for central metabolites analysis yet.

\section{Conclusion}

This study allows a better understanding of the differential engraftment and therapy adaptation patterns of leukemic cells, and provides several important foundations for further in vivo metabolomic studies. This study also highlight plasma metabolic signatures as potential biomarkers of metabolic status of blasts and of therapy resistance. 


\section{Abbreviations}

Page 13/25 


\begin{tabular}{|c|c|}
\hline 2.3-PG & 2.3-diphosphoglycerate \\
\hline 2-HG & 2-hydroxyglutarate \\
\hline 6-PG & 6-phosphogluconate \\
\hline ADP & Adenosine diphosphate \\
\hline aKG & Alpha-ketoglutarate \\
\hline Ala & Alanine \\
\hline AML & Acute myeloid leukemia \\
\hline AMP & Adenosine monophosphate \\
\hline AraC & Cytarabine \\
\hline Asn & Asparagine \\
\hline Asp & Aspartate \\
\hline ATP & Adenosine triphosphate \\
\hline BM & Bone marrow \\
\hline Cis-Aco & Cis-aconitate \\
\hline Cit \& IsoCit & Citrate and Isocitrate \\
\hline CLDX & Cell-line derived xenograft \\
\hline CMP & Cytosine monophosphate \\
\hline DHODH & Dihydroorotate dehydrogenase \\
\hline EMD & Extra-medullar disease \\
\hline ETC & Electron transport chain \\
\hline Fru6P & Fructose-6-phosphate \\
\hline FruBP & Frucose-1.6-biphosphate \\
\hline Fum & Fumarate \\
\hline GalN-1P & Galactosamine-1-phosphate \\
\hline GDP & Guanosine diphosphate \\
\hline Glc6P & Glucose-6-phosphate \\
\hline GICNAc-1P & $\mathrm{N}$-acetylglucosamine-1-phosphate \\
\hline GlcNAc-6P & $\mathrm{N}$-acetylglucosamine-6-phosphate \\
\hline Gln & Glutamine \\
\hline
\end{tabular}

Page 14/25 


\begin{tabular}{|c|c|}
\hline 2.3-PG & 2.3-diphosphoglycerate \\
\hline Glu & Glutamate \\
\hline Gly & Glycine \\
\hline Gly3P & Glycerol-3-phosphate \\
\hline GMP & Guanosine monophosphate \\
\hline His & Histidine \\
\hline IDMS & Isotope dilution method standard \\
\hline Ile & Isoleucine \\
\hline LC-MS & Liquid chromatography - Mass spectrometry \\
\hline Mal & Malate \\
\hline Man6P & Mannose-6-phosphate \\
\hline Met & Methionine \\
\hline MMMDB & Mouse multiple tissue metabolome database \\
\hline MSI & Mass spectrometry imaging \\
\hline NSG mouse & Non-obese diabetic scid gamma mouse \\
\hline OAA & Oxaloacetate \\
\hline Oro & Orotate \\
\hline OxPHOS & Oxidative phosphorylation \\
\hline P5P & Pentoses-5-phosphate \\
\hline PDX & Patient derived xenograft \\
\hline PEP & Phosphoenolpyruvate \\
\hline Phe & Phenylalanine \\
\hline Pro & Proline \\
\hline Pyr & Pyruvate \\
\hline Rib1P & Ribose-1-phosphate \\
\hline ROS & Reactive oxygen species \\
\hline Sed7P & Sedoheptulose-7-phosphate \\
\hline Ser & Serine \\
\hline SCM & Single cell metabolomics \\
\hline
\end{tabular}

Page 15/ 25 


\begin{tabular}{|ll|}
\hline 2.3-PG & 2.3-diphosphoglycerate \\
\hline SP & Spleen \\
\hline ssGSEA & Single sample gene set enrichment analysis \\
\hline Suc & Succinate \\
SWAT & Subcutaneous white adipose tissue \\
\hline TCA & Tricarboxylic acid \\
\hline Thr & Threonine \\
\hline Trp & Tryptophan \\
\hline Tyr & Tyrosine \\
UDP & Uridine diphosphate \\
UDP-AcGlcN & Uridine diphosphate-N-acetylglucosamine \\
\hline UMP & Uridine monophosphate \\
\hline UTP & Uridine triphosphate \\
\hline
\end{tabular}

\section{Declarations}

\section{Acknowledgements}

We thank Anne-Marie Benot, Muriel Serthelon and Stéphanie Nevouet for their daily help about the administrative and financial management of the Sarry lab. MetaTOUL (Metabolomics \& Fluxomics Facitilies, Toulouse, France, www.metatoul.fr) is part of the national infrastructure MetaboHUB-ANR-11INBS-0010 (The French National infrastructure for metabolomics and fluxomics, www.metabohub.fr).

\section{Funding}

This work was also supported by grants from the Laboratoire d'Excellence Toulouse Cancer (TOUCAN and TOUCAN2.0; contract ANR11-LABEX), the Canceropole GSO (MetaboCancerGSO), La Ligue Nationale de Lutte Contre le Cancer, the Fondation ARC, the Fondation Toulouse Cancer Santé and the Association G.A.E.L. MetaToul is supported by grants from the Région Midi-Pyrénées, the European Regional Development Fund, the SICOVAL, the Infrastructures en Biologie Santé et Agronomie (IBiSa, France), the Centre National de la Recherche Scientifique (CNRS) and the Institut National de la Recherche Agronomique (INRA). G.C. has a fellowship from the Ligue Nationale de Lutte Contre le Cancer.

\section{Ethics approval}

All animal studies described below were conducted in accordance with... 


\section{Consent for publication}

Not applicable

\section{Competing interests}

The authors declare that they have no competing interests.

\section{References}

1. Pascual, G. et al. Targeting metastasis-initiating cells through the fatty acid receptor CD36. Nature 541, 41-45 (2017).

2. Lien, E. C. et al. Glutathione biosynthesis is a metabolic vulnerability in $\mathrm{PI}(3) \mathrm{K} /$ Akt-driven breast cancer. Nat. Cell Biol. 18, 572-578 (2016).

3. Labuschagne, C. F., Cheung, E. C., Blagih, J., Domart, M.-C. \& Vousden, K. H. Cell Clustering Promotes a Metabolic Switch that Supports Metastatic Colonization. Cell Metab. 30, 720-734.e5 (2019).

4. Farge, T. et al. Chemotherapy-Resistant Human Acute Myeloid Leukemia Cells Are Not Enriched for Leukemic Stem Cells but Require Oxidative Metabolism. Cancer Discov. 7, 716-735 (2017).

5. Zacksenhaus, E. et al. Mitochondrial OXPHOS Induced by RB1 Deficiency in Breast Cancer: Implications for Anabolic Metabolism, Stemness, and Metastasis. Trends Cancer 3, 768-779 (2017).

6. Cruz-Bermúdez, A. et al. Cisplatin resistance involves a metabolic reprogramming through ROS and PGC-1 a in NSCLC which can be overcome by OXPHOS inhibition. Free Radic. Biol. Med. 135, 167181 (2019).

7. Sica, V., Pedro, J. M. B.-S., Stoll, G. \& Kroemer, G. Oxidative phosphorylation as a potential therapeutic target for cancer therapy. Int. J. Cancer 146, 10-17 (2020).

8. Roth, K. G., Mambetsariev, I., Kulkarni, P. \& Salgia, R. The Mitochondrion as an Emerging Therapeutic Target in Cancer. Trends Mol. Med. 26, 119-134 (2020).

9. Ye, H. et al. Subversion of Systemic Glucose Metabolism as a Mechanism to Support the Growth of Leukemia Cells. Cancer Cell 34, 659-673.e6 (2018).

10. Tabe, Y., Lorenzi, P. L. \& Konopleva, M. Amino acid metabolism in hematologic malignancies and the era of targeted therapy. Blood 134, 1014-1023 (2019).

11. Stuani, L., Sabatier, M. \& Sarry, J.-E. Exploiting metabolic vulnerabilities for personalized therapy in acute myeloid leukemia. BMC Biol. 17, (2019).

12. Mishra, D. \& Banerjee, D. Lactate Dehydrogenases as Metabolic Links between Tumor and Stroma in the Tumor Microenvironment. Cancers 11, (2019).

13. van Gastel, N. et al. Induction of a Timed Metabolic Collapse to Overcome Cancer Chemoresistance. Cell Metab. 32, 391-403.e6 (2020).

14. Marine, J.-C., Dawson, S.-J. \& Dawson, M. A. Non-genetic mechanisms of therapeutic resistance in cancer. Nat. Rev. Cancer 20, 743-756 (2020). 
15. Stuani, L. et al. Mitochondrial metabolism supports resistance to IDH mutant inhibitors in acute myeloid leukemia. J. Exp. Med. 218, (2021).

16. Akhaddar, A., Zyani, M., Mikdame, M. \& Boucetta, M. Acute myeloid leukemia with brain involvement (chloroma). Intern. Med. Tokyo Jpn. 50, 535-536 (2011).

17. Stölzel, F. et al. The prevalence of extramedullary acute myeloid leukemia detected by 18FDGPET/CT: final results from the prospective PETAML trial. Haematologica 105, 1552-1558 (2020).

18. $\mathrm{Ye} \mathrm{H}$, et al. Leukemic stem cells evade chemotherapy by metabolic adaptation to an adipose tissue niche. 27 (2017).

19. Ye, H. et al. The Hepatic Microenvironment Uniquely Protects Leukemia Cells through Induction of Growth and Survival Pathways Mediated by LIPG. Cancer Discov. 11, 500-519 (2021).

20. Mendes, N. et al. Animal Models to Study Cancer and Its Microenvironment. Adv. Exp. Med. Biol. 1219, 389-401 (2020).

21. Walsh, N. C. et al. Humanized Mouse Models of Clinical Disease. Annu. Rev. Pathol. 12, 187-215 (2017).

22. Olson, B., Li, Y., Lin, Y., Liu, E. T. \& Patnaik, A. Mouse Models for Cancer Immunotherapy Research. Cancer Discov. 8, 1358-1365 (2018).

23. Shultz, L. D., Brehm, M. A., Garcia-Martinez, J. V. \& Greiner, D. L. Humanized mice for immune system investigation: progress, promise and challenges. Nat. Rev. Immunol. 12, 786-798 (2012).

24. Saland, E. et al. A robust and rapid xenograft model to assess efficacy of chemotherapeutic agents for human acute myeloid leukemia. Blood Cancer J. 5, e297 (2015).

25. Tirado-Gonzalez, I. et al. CRISPR/Cas9-edited NSG mice as PDX models of human leukemia to address the role of niche-derived SPARC. Leukemia 32, 1049-1052 (2018).

26. Okada, S., Vaeteewoottacharn, K. \& Kariya, R. Establishment of a Patient-Derived Tumor Xenograft Model and Application for Precision Cancer Medicine. Chem. Pharm. Bull. (Tokyo) 66, 225-230 (2018).

27. Nanni, P. et al. Bone sarcoma patient-derived xenografts are faithful and stable preclinical models for molecular and therapeutic investigations. Sci. Rep. 9, 12174 (2019).

28. Layssol-Lamour CJ, Sarry J-E, Braun J-PD, Trumel C. \& Bourgès-Abella, N. H. Reference Values for Hematology, Plasma Biochemistry, Bone Marrow Cytology and Bone Histology of NOD.Cg-Prkdcscid II2rgtm1Wjl/SzJ Immunodeficient Mice. J Am Assoc Lab Anim Sci JAALAS. 2020. doi:10.30802/AALAS-JAALAS-20-000020.

29. Sugimoto, M. et al. MMMDB: Mouse Multiple Tissue Metabolome Database. Nucleic Acids Res. 40, D809-814 (2012).

30. Boyd, A. L. et al. Identification of Chemotherapy-Induced Leukemic-Regenerating Cells Reveals a Transient Vulnerability of Human AML Recurrence. Cancer Cell 34, 483-498.e5 (2018).

31. Blomme, A. et al. Murine stroma adopts a human-like metabolic phenotype in the PDX model of colorectal cancer and liver metastases. Oncogene 37, 1237-1250 (2018). 
32. Beaudry, P. et al. A Pilot Study on the Utility of Serum Metabolomics in Neuroblastoma Patients and Xenograft Models. Pediatr. Blood Cancer 63, 214-220 (2016).

33. Yao, T. \& Asayama, Y. Animal-cell culture media: History, characteristics, and current issues. Reprod. Med. Biol. 16, 99-117 (2017).

34. Lagziel, S., Gottlieb, E. \& Shlomi, T. Mind your media. Nat. Metab. 2, 1369-1372 (2020).

35. Vande Voorde, J. et al. Improving the metabolic fidelity of cancer models with a physiological cell culture medium. Sci. Adv. 5, eaau7314 (2019).

36. Cantor, J. R. et al. Physiologic Medium Rewires Cellular Metabolism and Reveals Uric Acid as an Endogenous Inhibitor of UMP Synthase. Cell 169, 258-272.e17 (2017).

37. Wu, L. et al. Quantitative analysis of the microbial metabolome by isotope dilution mass spectrometry using uniformly 13C-labeled cell extracts as internal standards. Anal. Biochem. 336, 164-171 (2005).

38. Scotland, S. et al. Mitochondrial energetic and AKT status mediate metabolic effects and apoptosis of metformin in human leukemic cells. Leukemia 27, 2129-2138 (2013).

39. Reich, M. et al. GenePattern 2.0. Nat. Genet. 38, 500-501 (2006).

40. Rohart, F., Gautier, B., Singh, A. \& Lê Cao, K.-A. mixOmics: An R package for 'omics feature selection and multiple data integration. PLoS Comput. Biol. 13, e1005752 (2017).

41. Madak, J. T., Bankhead, A., Cuthbertson, C. R., Showalter, H. D. \& Neamati, N. Revisiting the role of dihydroorotate dehydrogenase as a therapeutic target for cancer. Pharmacol. Ther. 195, 111-131 (2019).

42. Aroua, N. et al. Extracellular ATP and CD39 Activate cAMP-Mediated Mitochondrial Stress Response to Promote Cytarabine Resistance in Acute Myeloid Leukemia. Cancer Discov. 10, 1544-1565 (2020).

43. Sykes, D. B. et al. Inhibition of Dihydroorotate Dehydrogenase Overcomes Differentiation Blockade in Acute Myeloid Leukemia. Cell 167, 171-186.e15 (2016).

44. Horibata, S. et al. Heterogeneity in refractory acute myeloid leukemia. Proc. Natl. Acad. Sci. U. S. A. 116, 10494-10503 (2019).

45. Vick, B. et al. An advanced preclinical mouse model for acute myeloid leukemia using patients' cells of various genetic subgroups and in vivo bioluminescence imaging. PloS One 10, e0120925 (2015).

46. Chen, W.-L. et al. A distinct glucose metabolism signature of acute myeloid leukemia with prognostic value. Blood 124, 1645-1654 (2014).

47. Terkelsen, T. et al. High-throughput proteomics of breast cancer interstitial fluid: identification of tumor subtype-specific serologically relevant biomarkers. Mol. Oncol. 15, 429-461 (2021).

48. Duncan, K. D., Fyrestam, J. \& Lanekoff, I. Advances in mass spectrometry based single-cell metabolomics. The Analyst 144, 782-793 (2019).

49. Buchberger, A. R., Delaney, K., Johnson, J. \& Li, L. Mass Spectrometry Imaging: A Review of Emerging Advancements and Future Insights. Anal. Chem. 90, 240-265 (2018). 
50. Ferguson, C. N., Fowler, J. W. M., Waxer, J. F., Gatti, R. A. \& Loo, J. A. Mass Spectrometry-Based Tissue Imaging of Small Molecules. Adv. Exp. Med. Biol. 1140, 99-109 (2019).

Figures

A
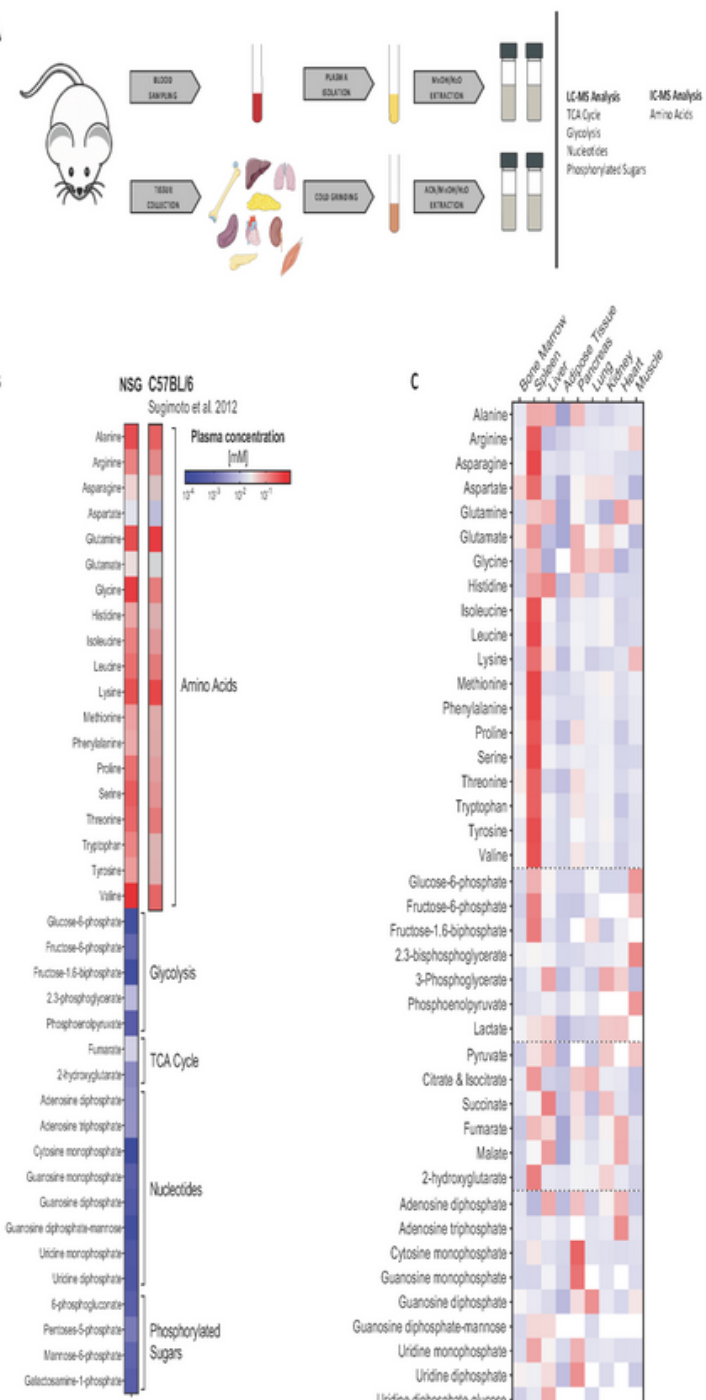

(

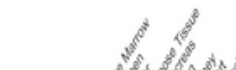

c
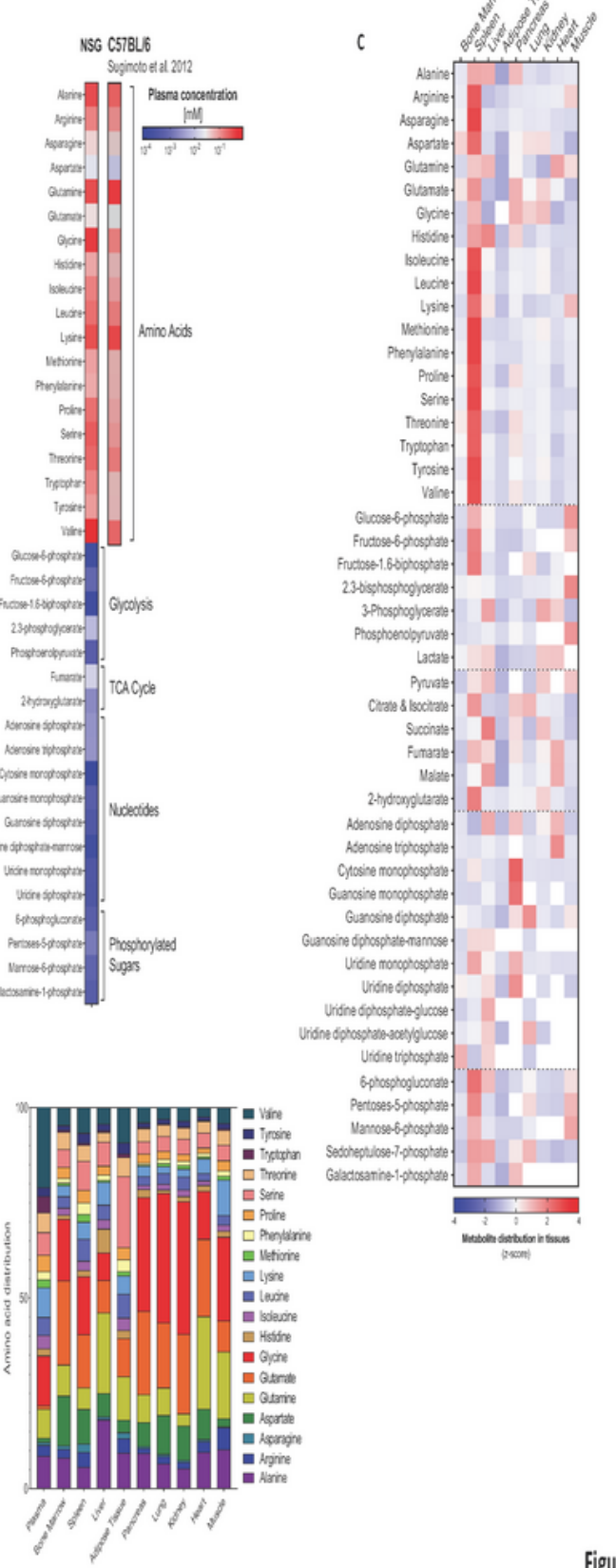

Figure1.

Figure 1 
Metabolic profiles of naïve immunodeficient NSG mice. (A) Experimental design and technical workflow. (B) Heatmap of the absolute concentrations of 38 metabolites in mice plasma. Amino acids were compared to C57BL/ 6 mice concentrations found in the MMMDB29 (C) Tissue distribution of the 56 studies metabolites, normalized as z-scores. (D) Tissue distribution of amino acids in each tissue analyzed in this study. Undetected metabolites are colored white. $n=3-4$.

A

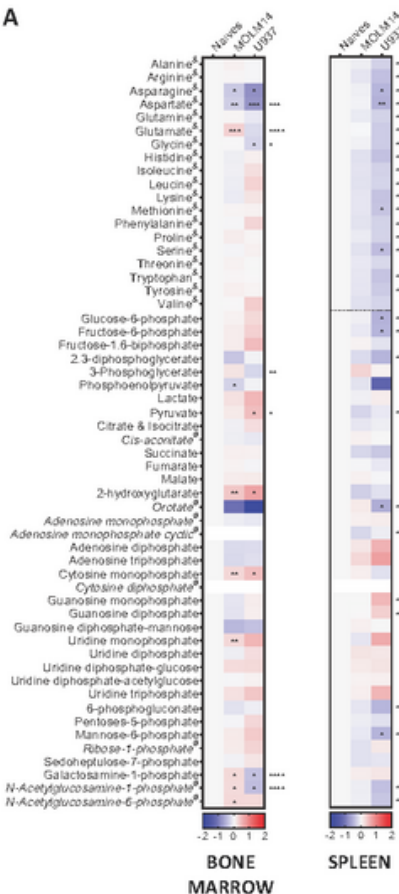

B

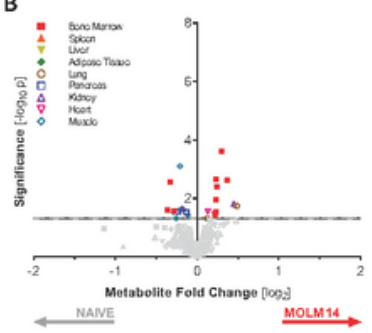

E
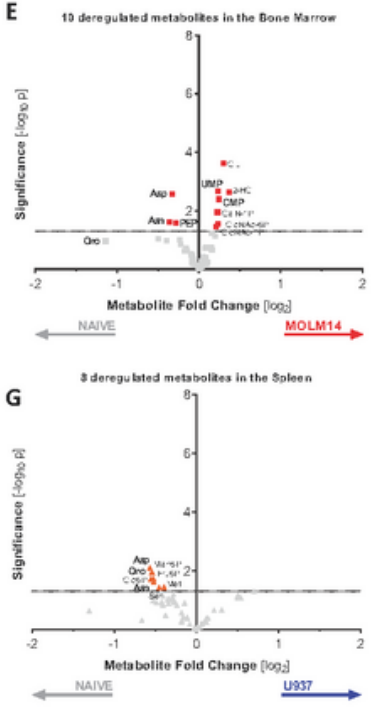
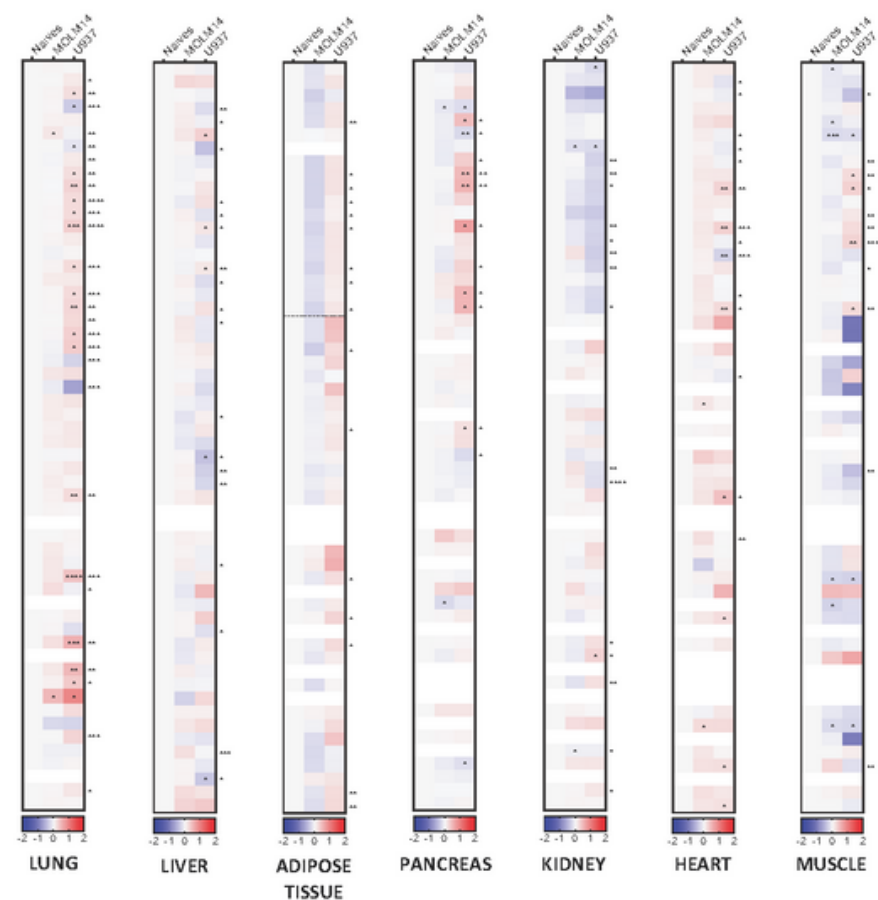

C

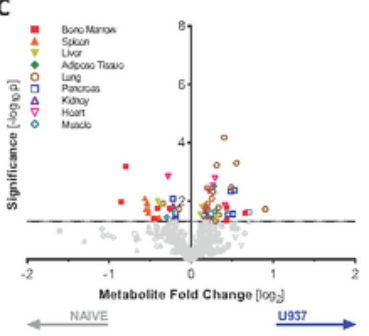

D
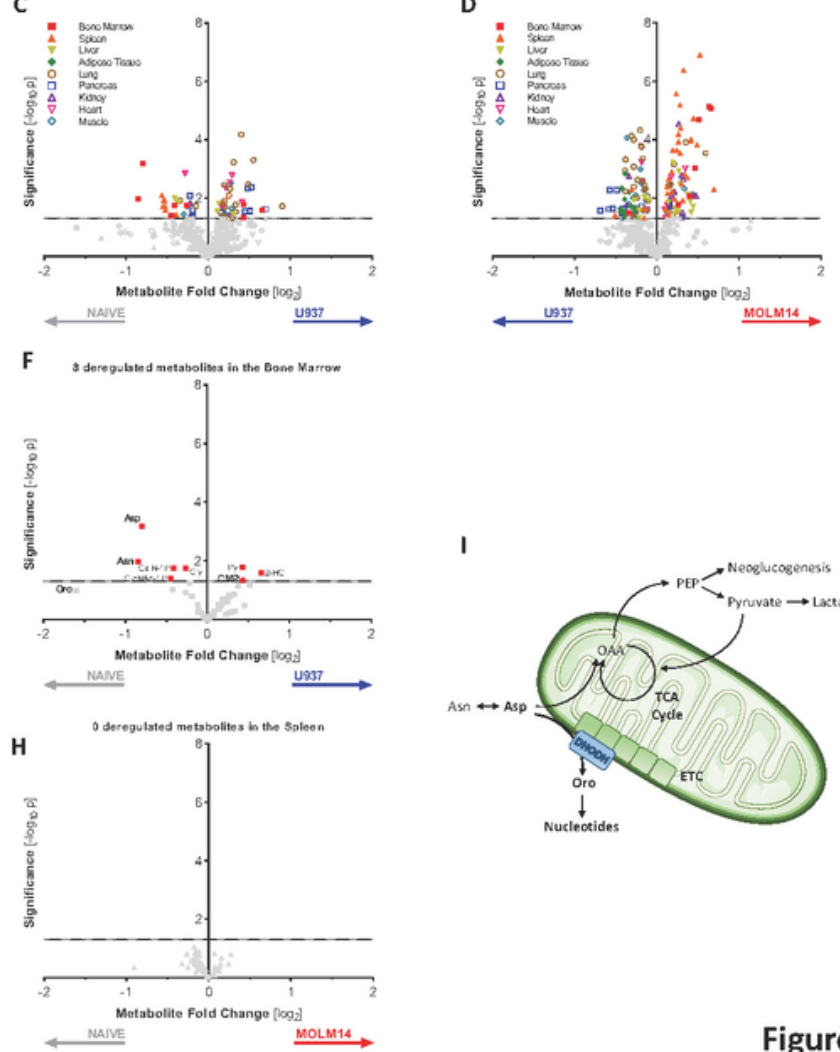

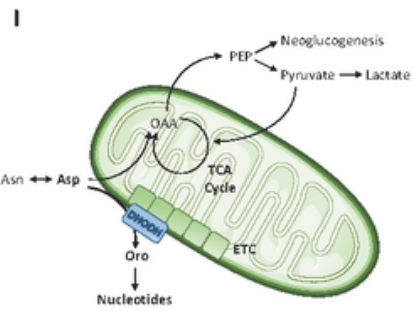

Figure 2.

Figure 2 
Cell Line Derived Xenograft (CLDX) models. (A) Experimental design. (B-C) Engraftment in the bone marrow and spleen of the two CLDX in millions of blasts (B) and percentage (C) of viable blasts. $n=5-10$, p-values calculated with Welsh statistical test.
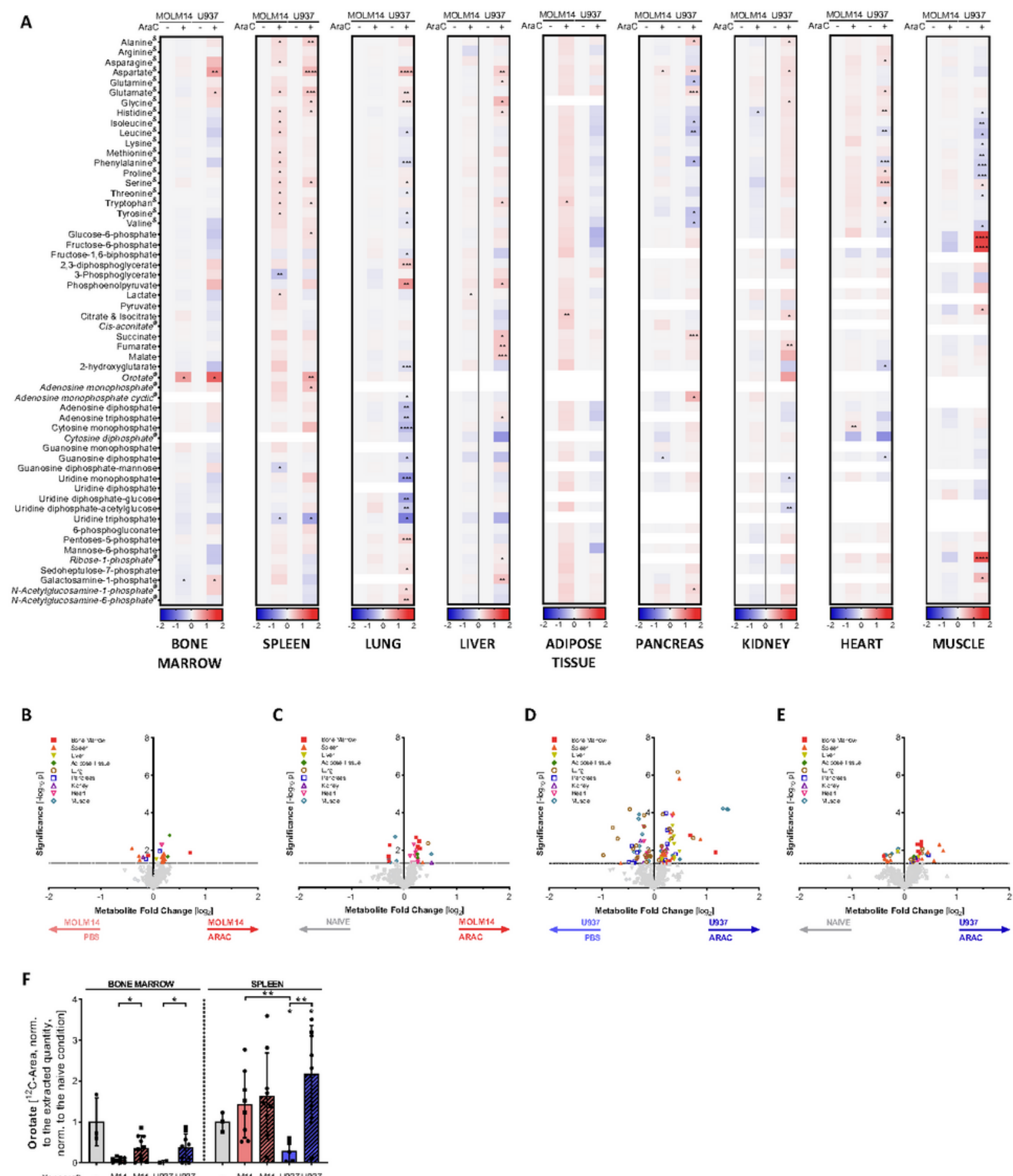

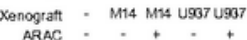

M14 M14 บ937U937

\section{Figure 3}

AML xenograft modulates tissue metabolic profiles. (A) Heatmap of the relative quantification of 56 metabolites in every solid tissue after MOLM14 or U937 xenograft (normalized to the naïve condition, 
$\log 10), \mathrm{p}$-values on the side show significance between the MOLM14 and U937 conditions. (B-C) Volcano plots showing the fold change (log2) ratio of all studied metabolites in every tissue of naïve vs. MOLM14 xenograft (B) and vs. U937 xenograft(C). (D) Volcano plot showing the fold change (log2) ratio of all studied metabolites in every tissue of the two xenografts. (E) Focus on the bone marrow metabolites modulations of the MOLM14 xenograft. (F) Focus on the bone marrow metabolites modulations of the U937 xenograft. (G) Focus on the spleen metabolites modulations of the U937 xenograft. (H) Aspartate metabolism is rewired in hematological tissues of xenografted mice. $n=4-10, p$-values calculated with Welsh statistical test. 
A

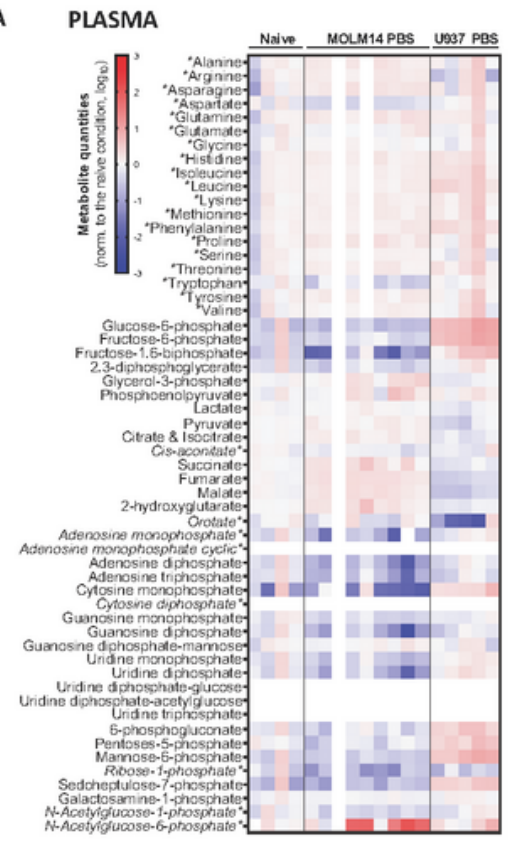

B

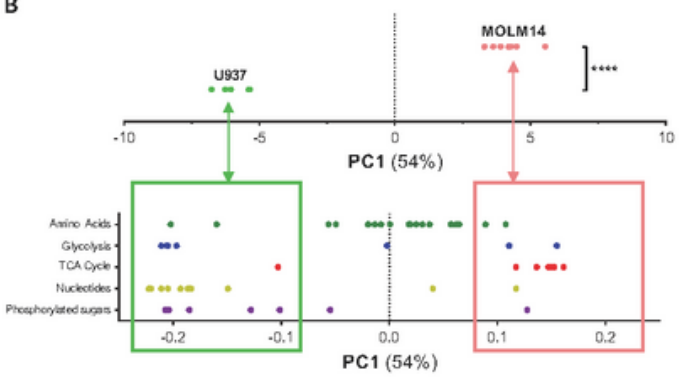

c

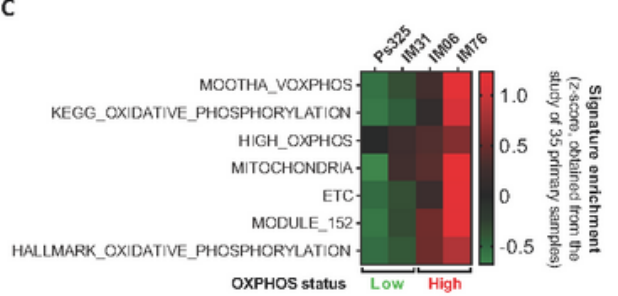

D
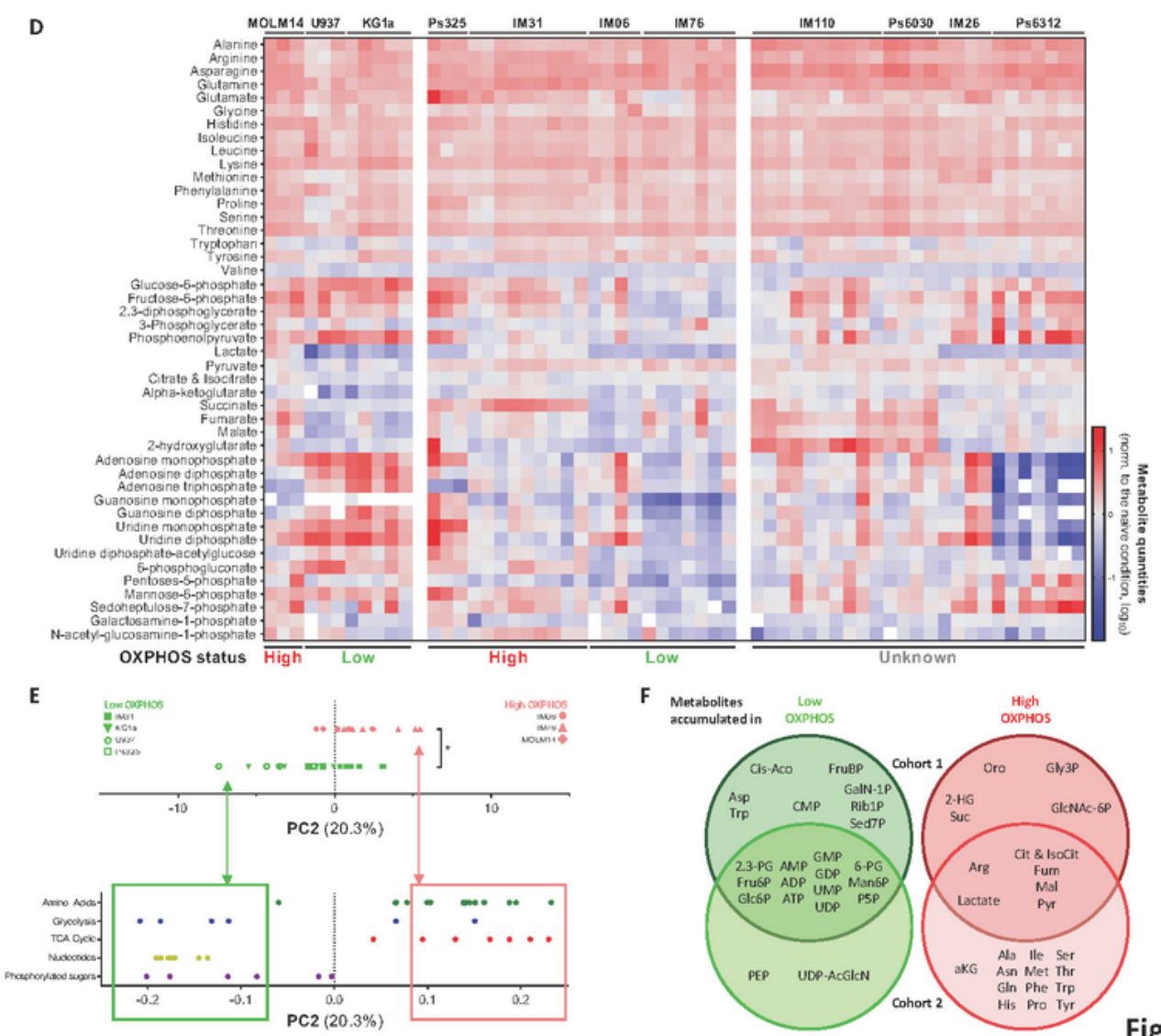

Figure 4.

\section{Figure 4}

Chemotherapy modulates tissue metabolic profiles of xenografted mice. (A) Heatmaps of the relative quantification of 56 metabolites in every solid tissue after AraC treatment in both xenografts (normalized to the control conditions, log10). (B-C) Volcano plots showing the fold change (log2) ratio of all studied metabolites in every tissue of MOLM14 xenografted mice, treated with PBS vs. ARAC (B), and the comparison of the naïve state with the residual disease (C). (D-E) Volcano plots showing the fold change 
(log2) ratio of all studied metabolites in every tissue of U937 xenografted mice, treated with PBS vs. ARAC (D), and the comparison of the naïve state with the residual disease (E). (F)Relative quantities of orotate in the bone marrow and the spleen; Metabolite\&: Amino Acid method, metabolite\#: 12Cmetabolites areas, others: $12 \mathrm{C} / 13 \mathrm{C}$-metabolites ratios; $n=4-10, p$-values calculated with Welsh statistical test.

\section{Image not available with this version}

\section{Figure 5}

Plasma metabolic profiles unravel cell characteristic signatures and potential biomarkers. (A) Heatmap of the relative concentrations of 56 metabolites in the plasma (normalized to the naïve condition, log2); $n=4$ 10. (B) PCA of all plasmas, representation of the first component, separating MOLM14 and U937 xenografted mice without treatment. (C) Heatmap of single sample Gene Set Enrichment Analysis (SSGSEA) of 4 primary samples. (D) Heatmaps of the relative concentration of 56 metabolites in the plasmas of 11 new cell line-derived xenografts (CLDX) and patient-derived xenografts (PDX) (normalized to the naïve condition, log2); n=3-10. (E) PCA of plasmas of mice xenografted with cell lines and primary samples with known OXPHOS status, representation of the second component, separating xenografted mice without treatment depending on their OXPHOS status. $(F)$ Venn diagrams of representative metabolites of low (green) and high (red) OXPHOS status in the two cohorts. p-values calculated with Welsh statistical test.

\section{Supplementary Files}

This is a list of supplementary files associated with this preprint. Click to download.

- Cognetetal2021SupplementaryFigures.pdf 